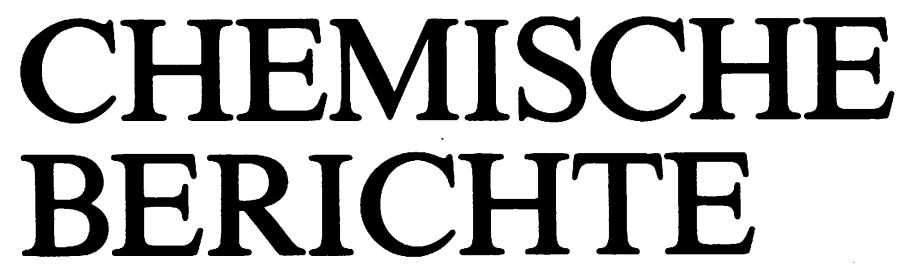

GEGRUNDET 1868

115. JAHRGANG

HERAUSGEGEBEN IM AUFTRAG DER

GESELLSCHAFT DEUTSCHER CHEMIKER

VON

K. HAFNER - W. KIRMSE - H. MUSSO - H. NÖTH • J. SAUER - E. WINTERFELDT

UNTER MITWIRKUNG VON

H. A. BRUNE - W. LÜTTKE - G. SPITELLER

REDAKTION: H. ZAHN

mit H. SCHILL, J. STREHLOW und A. WIELAND 


\section{BEMERKUNGEN DER REDAKTION}

1. Die Chemischen Berichte (zu zitieren als Chem. Ber.) setzen die Berichte der Deutschen Chemischen Gesellschaft (zu zitieren als Ber. Dtsch. Chem. Ges.) fort.

2. Die „Berichte“ enthalten Originalmitteilungen aus allen Gebieten der Chemie.

3. Die „Berichte“ erscheinen monatlich; ein Registerheft beschließt jeden Jahrgang.

4. Die Verantwortung für ihre Mitteilungen tragen die Verfasser selbst. - Der Korrespondenzautor ist durch einen hochgestellten Stern hervorgehoben. Die Zugehörigkeit der Autoren zu den Instituten ist durch hochgestellte Kleinbuchstaben markiert.

5. Es werden grundsätzlich nur Arbeiten aufgenommen, die vorher weder im Inland noch im Ausland veröffentlicht worden sind.

6. Eine Anweisung zur Abfassung von Manuskripten für die „Berichte“ wird auf Wunsch zugestellt.

7. Manuskripte sind zu senden an Redaktion der Chemischen Berichte. Dr. H. Zahn, Am Klopferspitz, D-8033 Martinsried, Telefon (089) 8585830.

8. Der Eingang der Abhandlungen wird den Autoren am Tage der Registrierung angezeigt.

9. Es werden nur Manuskripte in deutscher oder englischer Sprache aufgenommen. Allen Beiträgen ist eine knappe Zusammenfassung (summary) in beiden Sprachen voranzustellen, einschließlich Titel in der jeweils zweiten Sprache.

10. Der Autor muß das alleinige Urheberrecht besitzen. Mit der Annahme des Manuskriptes durch die Redaktion überträgt er dem Verlag Chemie das ausschließliche Nutzungsrecht, insbesondere das Recht der Vervielfältigung wie Fotokopie, Mikrofilm - oder mit irgendeinem anderen Verfahren - oder das Manuskript in eine von Maschinen, insbesondere von Datenverarbeitungsmaschinen, verwendbare Sprache zu übergeben oder zu übersetzen (auch in fremde Sprachen).

11. Den Autoren werden 75 Sonderdrucke unentgeltlich portofrei zugesandt. Wünscht ein Autor mehr als 75 Abzüge, so ist dies auf dem Manuskript oder spätestens bei Rücksendung der Korrektur auf dieser zu vermerken. Den Autoren werden nur die Selbstkosten für die Zahl der die Freiexemplare überschreitenden Sonderabzüge berechnet.

12. Anfragen nach dem Verbleib nicht eingetroffener Berichte-Hefte oder Sonderdrucke sind zu richten an: Verlag Chemie GmbH, Pappelallee 3, Postfach 1260/1280, D-6940 Weinheim, Telefon (06201) 602-1.

Die Chemischen Berichte erscheinen monatlich. Der Jahresbezugspreis betrăgt 670. - DM zuzügl. Versandgebühren. Einzelheft 76. - DM. In diesem Preis ist die Mehrwertsteuer enthalten. Die Bezugsbedingungen für Mitglieder der Gesellschaft Deutscher Chemiker werden auf Anfrage von der Geschäftsstelle, Carl-Bosch-Haus, Varrentrappstraße 40-42, Postfach 900440, D-6000 Frankfurt 90, mitgeteilt. - Bestellungen richten Sie bitte an Ihre Fachbuchhandlung oder unmittelbar an den Verlag. - Abbestellungen nur bis spătestens 2 Monate vor Ablauf des Kalenderjahres. - Die Lieferung erfolgt auf Rechnung und Gefahr des Empfängers. Gerichtsstand und Erfüllungsort: Weinheim/Bergstr.

Verlag und Anzeigenabteilung: Verlag Chemie GmbH, Pappelallee 3, Post fach 1260/1280, D-6940 Weinheim. Fernsprecher (06201) 602-1, Fernschreiber 465516 vchwh d.

\section{For USA and Canada}

Published monthly by Verlag Chemie, GmbH, Weinheim, Federal Republic of Germany, Annual subscription price: $\$ 455.00$ including postage. Second-class postage paid at Jamaica, N. Y. Printed in the Federal Republic of Germany. Air-freighted and mailed in the United States by Publications Expediting Inc., 200 Meacham Avenue, Elmont, N. Y. 11003. Subscribers in North America should place their order through Verlag Chemie International Inc., Plaza Centre, Suite E, 1020 N. W. Sixth Street, Deerfield Beach, Florida 33441.

U.S. A Postmasters: Send address changes for Chemische Berichte to Verlag Chemie International Inc., Plaza Centre, Suite E, 1020 N.W. 6th Street, Deerfield Beach, FL 33441. 
115. Jahrgang 1982

\section{ANORGANISCHE CHEMIE}

Fischer Helmut, Fischer Ernst Otto und Cai Ruifang: Übergangsmetall-Carben-Komplexe, CXXII; Kinetische und mechanistische Untersuchungen von ÜbergangsmetallKomplex-Reaktionen, XII: Pentacarbonyl[diethylamino(triphenylplumbyl)carben]chrom und trans-Tetracarbonyl(diethylaminocarbin)(triphenylplumbyl)chrom: Präparative Untersuchungen und Kinetik der $\mathrm{C}, \mathrm{Cr}$-Wanderung von $\mathrm{PbPh}_{3} \ldots \ldots \ldots$

Roesky Herbert W., Schmieder Wilfried, Isenberg Wilhelm, Sheldrick William S. und Sheldrick George M.: Schwefel-Anionen mit der Koordinationszahl 3: Synthese,

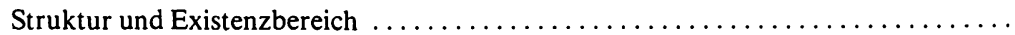

Paetzold Peter und Plotho von Christoph: Über weitere monomere Borimide und ihre

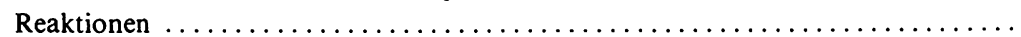

Leidinger Walter und Sundermeyer Wolfgang: Zur Chemie des (Trifluormethyl)sulfinylamins, $\mathrm{CF}_{3} \mathrm{NSO}$, und verwandter Verbindungen $\ldots \ldots \ldots \ldots \ldots \ldots \ldots \ldots \ldots$

Werner Helmut, Kraus Hans-Jürgen, Schubert Ulrich und Ackermann Klaus: Untersuchungen zur Reaktivität von Metall- $\pi$-Komplexen, XXXVI: Zweikernige Sandwichkomplexe mit Metall-Metall-Bindung: Synthese und Struktur von $\left(\mu-\mathrm{C}_{5} \mathrm{H}_{5}\right)_{2} \mathrm{Pd}_{2}$ -

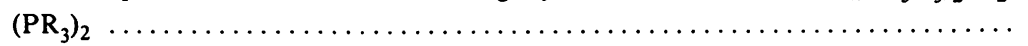

Werner Helmut, Kraus Hans-Jürgen und Thometzek Peter: Untersuchungen zur Reaktivität von Metall- $\pi$-Komplexen, XXXVII: Brückenaustauschreaktionen an zweikernigen $\mu$-(Cyclopentadienyl)-dipalladium-Komplexen: Synthese sandwich-artiger heterometallischer $\mathrm{Pd}_{2} \mathrm{Cr}$ - und $\mathrm{Pd}_{2}$ Mo-Cluster $\ldots \ldots \ldots \ldots \ldots \ldots \ldots \ldots \ldots \ldots$

Henle Hartmut und Mews Rüdiger: Aminoschwefeldifluorid-Salze ..............

\section{ORGANISCHE CHEMIE}

Schill Gottfried, Beckmann Wolfgang und Fritz Hans: Synthese eines [3]-Praecatenans . .

Griesbaum Karl und Neumeister Joachim: Ozonolyse von symmetrisch 1,2-disubstituierten Ethylenen in salzsaurem Methanol: Säurekatalysierte Folgereaktionen von Primärspaltprodukten

Frauenrath Herbert, Runsink Jan und Scharf Hans-Dieter: Carboxonium-Ionen-Reaktionen cyclischer Acetale, VI: Der sterische Verlauf der säurekatalysierten Umlagerung von 4,5-Dihydro-1,3-dioxepinen zu Tetrahydrofuran-3-carbaldehyden .......

Kirmse Wolfgang und Hellwig Georg: Desaminierungsreaktionen, 37: Zerfall von Bicyclo[6.1.0]nonan- und Bicyclo[6.1.0]non-2-en-9-diazonium-Ionen ..............

Schaumann Ernst: Thioketen-Synthesen, VI: Stabile Thioketene durch Schwefelung ste-

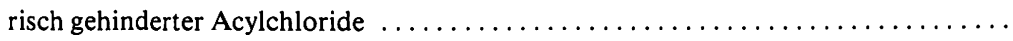

Effenberger Franz, Maier Roland, Schönwälder Karl-Heinz und Ziegler Thomas: Enolether, XIII: Die Acylierung von Enolethern mit reaktiven Carbonsäurechloriden ... 
El-Farargy Ahmed F., Lehnig Manfred und Neumann Wilhelm P.: Uber sterisch gehinderte freie Radikale, IX: Zur Gewinnung und Struktur von Stannylradikalen $\mathbf{R}_{\mathbf{3}} \mathbf{S n}$.

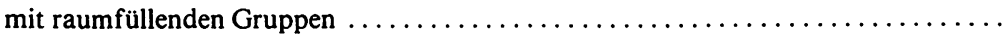

Vilsmaier Elmar, Klein Claus Michael und Tröger Wolfgang: Enaminosulfonium-Salze als Basis für stereoselektive Synthesen von Aminobicyclo[n.1.0]alkanen ..........

Metz Hans-Joachim und Neunhoeffer Hans: Zur Synthese von 1,2,4-Triazinen, IX:

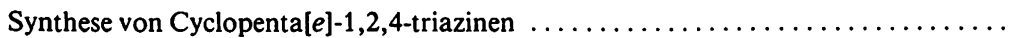

Frenking Gernot, Hülskämper Ludwig und Weyerstahl Peter: Reaktivität von 1-Cyclopropen-1-carbonsäurelactonen in Abhängigkeit von der Größe des Lactonringes . . . .

Hanack Michael, Metz Josef und Pawlowski Georg: Lösliche trans-Di-1-alkinyl- und Poly-trans-ethinyl(tetraalkylphthalocyaninato)metall-IVB-Derivate ...........

Heesing Albert und Steinkamp Heinz: Mechanismen bei elektrophilen Reaktionen von reaktiven Stickstoffverbindungen mit Phosphanen .....................

Gessner Uwe, Heesing Albert, Keller Ludwig und Kleine Homann Walter: Zur Beweglichkeit im Ionenpaar bei der aromatischen Umlagerung von $O$-Sulfonyl- $N$-phenyl-

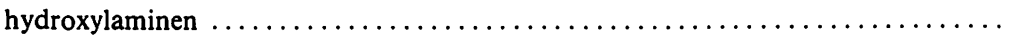

Jackman Lloyd M., Dunne Theresa S., Müller Bodo und Quast Helmut: Konformation von Tetraaryl-3,7-diazabicyclo[3.3.1]nonanen und Tetra- und Pentaaryl-1,3-diazaadamantanen in Lösung. Eine NMR-Studie $\ldots \ldots \ldots \ldots \ldots \ldots \ldots \ldots \ldots \ldots \ldots$

Neidlein Richard, Tran-Viet Dao, Gieren Alfred, Kokkinidis Michail, Wilckens Rudolf, Geserich Hans-Peter und Ruppel Wolfgang: Synthesen, Konstitutionsaufklärung, Röntgenstrukturanalyse und elektrische Eigenschaften von Di- und Polychalkogen-

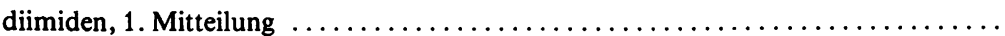

Rademacher Andreas, Märkle Suse und Langhals Heinz: Lösliche Perylen-Fluoreszenz-

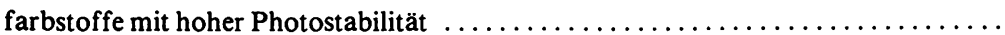

Willms Lothar, Günther Dieter und Hüttelmaier Thomas: Darstellung und Umsetzungen von 1,2-Dihalogenalkylsulfonylisocyanaten $\ldots \ldots \ldots \ldots \ldots \ldots \ldots \ldots \ldots \ldots$

Böhme Horst, Denis Jean-Pierre und Kämpchen Thomas: Reaktionsprodukte aus $N, N$ Bis(chlormethyl)carboxamiden und Diaziridinen $\ldots \ldots \ldots \ldots \ldots \ldots \ldots \ldots$ 


\section{INORGANIC CHEMISTRY}

Fischer Helmut, Fischer Ernst Otto, and Cai Ruifang: Transition Metal Carbene Complexes, CXXII; Kinetic and Mechanistic Investigations of Transition Metal Complex Reactions, XII: Pentacarbonyl[diethylamino(triphenylplumbyl)carbene]chromium and trans-Tetracarbonyl(diethylaminocarbyne)(triphenylplumbyl)chromium: Preparative Investigations and Kinetics of the $\mathrm{C}, \mathrm{Cr}$-Migration of $\mathrm{PbPh}_{3} \ldots \ldots \ldots \ldots \ldots$

Roesky Herbert W., Schmieder Wilfried, Isenberg Wilhelm, Sheldrick William S., and Sheldrick George M: Anions of Sulfur with the Coordination Number 3: Synthesis,

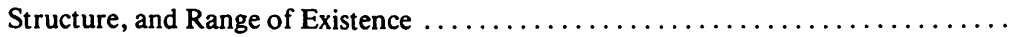

Paetzold Peter and Plotho von Christoph: New Monomeric Boron Imides and Their

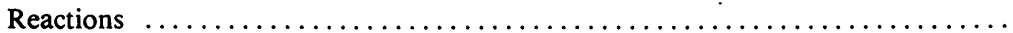

Leidinger Walter and Sundermeyer Wolfgang: On the Chemistry of (Trifluoromethyl)sulfinylamine, $\mathrm{CF}_{3} \mathrm{NSO}$, and Related Compounds $\ldots \ldots \ldots \ldots \ldots \ldots \ldots \ldots$

Werner Helmut, Kraus Hans-Jürgen, Schubert Ulrich, and Ackermann Klaus: Studies on the Reactivity of Metal $\pi$-Complexes, XXXVI: Dinuclear Sandwich Complexes Containing a Metal-Metal Bond: Synthesis and Structure of $\left(\mu-\mathrm{C}_{5} \mathrm{H}_{5}\right)_{2} \mathrm{Pd}_{2}\left(\mathrm{PR}_{3}\right)_{2} \ldots$

Werner Helmut, Kraus Hans-Jürgen, and Thometzek Peter: Studies on the Reactivity of Metal $\pi$-Complexes, XXXVII: Bridge-Exchange Reactions with Dinuclear $\mu$-(Cyclopentadienyl)-dipalladium Complexes: Synthesis of Sandwich-like Heterometallic

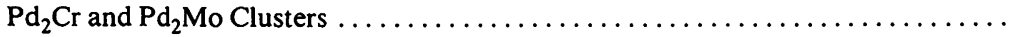

Henle Hartmut and Mews Rüdiger: Aminosulfur Difluoride Salts $\ldots \ldots \ldots \ldots \ldots \ldots$

\section{ORGANIC CHEMISTRY}

Schill Gottfried, Beckmann Wolfgang, and Fritz Hans: Synthesis of a [3]-Precatenane ...

Griesbaum Karl and Neumeister Joachim: Ozonolysis of Symmetrically 1,2-Disubstituted Ethylenes in $\mathrm{HCl} /$ Methanol Solutions: Acid Catalyzed Reactions of Primary Cleavage Products

Frauenrath Herbert, Runsink Jan, and Scharf Hans-Dieter: Reactions of Cyclic Acetale Carboxonium Ions, VI: The Stereoselectivity of the Acid-catalysed Rearrangement of 4,5-Dihydro-1,3-dioxepins to Tetrahydrofuran-3-carbaldehydes $\ldots \ldots \ldots \ldots \ldots$.

Kirmse Wolfgang and Hellwig Georg: Deamination Reactions, 37: Decomposition of Bicyclo[6.1.0]nonane- and Bicyclo[6.1.0]non-2-ene-9-diazonium Ions

Schaumann Ernst: Thioketene Syntheses, VI: Stable Thioketenes via Thionation of Sterically Hindered Acyl Chlorides

Effenberger Franz, Maier Roland, Schönwälder Karl-Heinz, and Ziegler Thomas: Enol Ethers, XIII: Acylation of Enol Ethers with Reactive Carbonyl Chlorides ........

El-Farargy Ahmed F., Lehnig Manfred, and Neumann Wilhelm P.: Sterically Hindered Free Radicals, IX: Generation and Structure of Stannyl Radicals $\mathrm{R}_{\mathbf{3}} \mathrm{Sn}^{\bullet}$ with Bulky

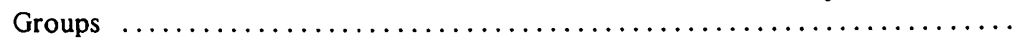


Vilsmaier Elmar, Klein Claus Michael, and Tröger Wolfgang: Enaminosulfonium Salts, Starting Materials for a Stereoselective Synthesis of Aminobicyclo[n.1.0]alkanes ...

Metz Hans-Joachim and Neunhoeffer Hans: Synthesis of 1,2,4-Triazines, IX: Synthesis

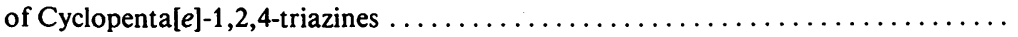

Frenking Gernot, Hülskämper Ludwig, and Weyerstahl Peter: Reactivity of 1-Cyclopropene-1-carboxylic Acid Lactones Dependent on the Size of the Lactone Ring ....

Hanack Michael, Metz Josef, and Pawlowski Georg: Soluble trans-Di-1-alkynyl- and Poly-trans-ethynyl(tetraalkylphthalocyaninato)metal IVB Derivatives $\ldots \ldots \ldots \ldots$

Heesing Albert and Steinkamp Heinz: Mechanisms in Electrophilic Reactions of Reactive

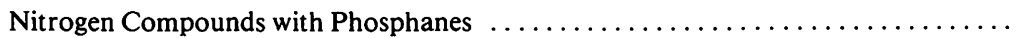

Gessner Uwe, Heesing Albert, Keller Ludwig, and Kleine Homann Walter: Internal Mobility in the Ion Pairs during Aromatic Rearrangement of $O$-Sulfonyl- $N$-phenylhydroxylamines

Jackman Lloyd M., Dunne Theresa S., Müller Bodo, and Quast Helmut: Conformation in Solution of Tetraaryl-3,7-diazabicyclo[3.3.1]nonanes and Tetra- and Pentaaryl1,3-diazaadamantanes. A Nuclear Magnetic Resonance Study ................

Neidlein Richard, Tran-Viet Dao, Gieren Alfred, Kokkinidis Michail, Wilckens Rudolf, Geserich Hans-Peter, and Ruppel Wolfgang: Syntheses, Structures, X-Ray Structure Analyses, and Electric Properties of Di- and Polychalkogen Diimides, Part 1 ......

Rademacher Andreas, Märkle Suse, and Langhals Heinz: Soluble Perylene Fluorescent

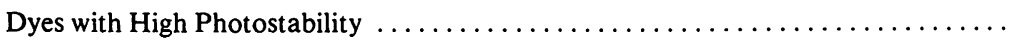

Willms Lothar, Günther Dieter, and Hüttelmaier Thomas: Synthesis and Reactions of

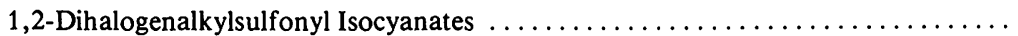

Böhme Horst, Denis Jean-Pierre, and Kämpchen Thomas: Reaction Products from $N, N$ Bis(chloromethyl)carboxamides and Diaziridines $\ldots \ldots \ldots \ldots \ldots \ldots \ldots \ldots$ 
Ackermann, K. s. Werner, H. . . . . . 2905

Beckmann, W. s. Schill, $G$.

2683

Böhme, H., Denis, J.-P. und

Kämpchen, $T$. 2947

Cai, R. s. Fischer, $H$.

Denis, J.-P. s. Böhme, $H$.

Dunne, T. S. s. Jackman, L. M. 2872

Effenberger, $F$., Maier, $R$.,

Schönwälder, K.-H. und

Ziegler, $T$. 2766

El-Farargy, A. F., Lehnig, M. und

Neumann, W. $P$. 2783

Fischer, E. O. s. Fischer, $H$. 2707

Fischer, H., Fischer, E. O. und Cai, R. . 2707

Frauenrath, $H$., Runsink, J. und

Scharf, H.-D. 2728

Frenking, G., Hülskämper, L. und

Weyerstahl, $P$. 2826

Fritz, H. s. Schill, G. 2683

Geserich, H.-P. s. Neidlein, R. 2898

Gessner, U., Heesing, A., Keller, L. und

Kleine Homann, $W$. 2865

Gieren, $A$. s. Neidlein, $R$. 2898

Griesbaum, K. und Neumeister, J. . . . . 2697

Günther, D. s. Willms, $L$. 2943

Hanack, M., Metz, J. und

Pawlowski, G. 2836

Heesing, A. und Steinkamp, $H$. 2854

- s. Gessner, $U$. 2865

Hellwig, G. s. Kirmse, $W$. 2744

Henle, $H$. und Mews, $R$. 2935

Hülskämper, L. s. Frenking, G. . . . . . 2826

Hüttelmaier, T. s. Willms, L. . . . . . . 2943

Isenberg, W. s. Roesky, H. W.

2714

Jackman, L. M., Dunne, T. S.,

Müller, $B$. und Quast, $H$ 2872

Kämpchen, T. s. Böhme, $H$.

Keller, L. s. Gessner, U. . . . . . . . . 2865

Kirmse, W. und Hellwig, G. . . . . . . 2744

Klein, C. M. s. Vilsmaier, E.
Kleine Homann, W. s. Gessner, U. . . 2865 Kokkinidis, M. s. Neidlein, $R . \quad \ldots \ldots \ldots 2898$ Kraus, H.-J. s. Werner, H. . . . . 2905, 2914 Langhals, H. s. Rademacher, A. . . . . 2927 Lehnig, M. s. El-Farargy, A. F. . . . . 2783 Leidinger, $W$. und Sundermeyer, $W . \ldots 2892$ Märkle, S. s. Rademacher, A. . . . . . 2927

Maier, R. s. Effenberger, F. . . . . . 2766

Metz, H.-J. und Neunhoeffer, H. . . . 2807

Metz, J. s. Hanack, M. .......... 2836

Mews, R. s. Henle, H. ......... 2935

Müller, B. s. Jackman, L. M. . . . . . . 2872

Neidlein, R., Tran-Viet, D., Gieren, A.,

Kokkinidis, M., Wilckens, R.,

Geserich, H.-P. und Ruppel, W. . 2898

Neumann, W. P. s. El-Farargy, A. F. . 2783

Neumeister, J. s. Griesbaum, K. . . . . . 2697

Neunhoeffer, H. s. Metz, H.-J. . . . . 2807

Paetzold, P. und Plotho, von, C. ..... 2819

Pawlowski, G. s. Hanack, M. . . . . . 2836

Plotho, von, C. s. Paetzold, P. . . . . . 2819

Quast, H. s. Jackman, L. M. . . . . . . 2872

Rademacher, A., Märkle, S. und

Langhals, $H$.

Roesky, H. W., Schmieder, W.,

Isenberg, $W .$, Sheldrick, $W . S$. und

Sheldrick, G. M. . . . . . . . . 2714

Runsink, J. s. Frauenrath, H. . . . . . 2728

Ruppel, W. s. Neidlein, $R$. . . . . . . 2898

Scharf, H.-D. s. Frauenrath, H. . . . . 2728

Schaumann, E. ............ 2755

Schill, G., Beckmann, W. und Fritz, H. 2683

Schmieder, W. s. Roesky, H. W. . . . 2714

Schönwälder, K.-H. s. Effenberger, F. . 2766

Schubert, U. s. Werner, H. . . . . . . 2905

Sheldrick, G. M. s. Roesky, H. W. . . . 2714

Sheldrick, W. S. s. Roesky, H. W. . . . 2714

Steinkamp, H. s. Heesing, A. . . . . . . 2854

Sundermeyer, W. s. Leidinger, W. . . . . 2892

Thometzek, P. s. Werner, H. ....... 2914 
Tran-Viet, D. s. Neidlein, R. ......2898 -, Kraus, H.-J. und Thometzek, P. .. 2914

Tröger, W. s. Vilsmaier, E. ....... $2795 \quad$ Weyerstahl, P. s. Frenking, G. . . . . 2826

Vilsmaier, E., Klein, C. M. und

Wilckens, $R$. s. Neidlein, $R . \ldots \ldots \ldots 2898$ Tröger, $W . \ldots \ldots \ldots \ldots \ldots \ldots$ 279

Werner, H., Kraus, H.-J., Schubert, $U$.

Willms, L., Günther, $D$. und

Hüttelmaier, $T$. ........... 2943

und Ackermann, $K$.

2905 Ziegler, T. s. Effenberger, F.

2766 
Chem. Ber. 115, $2927-2934$ (1982)

\title{
Lösliche Perylen-Fluoreszenzfarbstoffe mit hoher Photostabilität
}

\author{
Andreas Rademacher ${ }^{1)}$, Suse Märkle ${ }^{2)}$ und Heinz Langhals* \\ Chemisches Laboratorium der Universität Freiburg, \\ Albertstr. 21, D-7800 Freiburg i. Br.
}

Eingegangen am 14. Dezember 1981

Die Darstellung einer Reihe von 3,4,9,10-Perylentetracarbonsäurediimiden 1 wird beschrieben und deren Lichtechtheit quantitativ untersucht und diskutiert. Es läßt sich zeigen, daß durch Einführung von tert-Butyl-Substituenten die als sehr schwerlöslich bekannten Perylen-Pigmentfarbstoffe in organischen Solventien leicht löslich werden und mit hohen Quantenausbeuten fluoreszieren.

\section{Soluble Perylene Fluorescent Dyes with High Photostability}

The preparation of several 3,4,9,10-perylenebis(dicarboximides) 1 is described and their photostability quantitatively determined and discussed. It is shown, that substitution of the insoluble perylene dye pigments with tert-butyl groups causes solubility in organic solvents leading to high quantum yields of these dyes.

Fluoreszenzfarbstoffe mit extrem großer Photostabilität (Lichtechtheit) werden u. a. für die Gewinnung von Solarenergie benötigt ${ }^{3)}$, so z. B. für den von Götzberger und Greubel ${ }^{4)}$ sowie Garvin $^{5)}$ entwickelten Fluoreszenz-Solarkollektor.

Es ist bekannt ${ }^{6,7)}$, daß die Perylenfarbstoffe 1, die 3,4,9,10-Perylentetracarbonsäurediimide, sehr lichtechte, fluoreszierende ${ }^{10)}$ Pigmente bilden. Da für den Fluoreszenzkollektor jedoch nur lösliche Farbstoffe verwendbar sind ${ }^{3}$ ), ist zu prüfen, inwieweit durch geeignete Substituenten $R$ in 1 Farbstoffe erhalten werden können, die in organischen Lösungsmitteln gut löslich sind und mit hohen Quantenausbeuten fluoreszieren. In anderem Zusammenhang ${ }^{3,8,9)}$ wurde gefunden, da 3 eine Substitution von Aromaten mit tert-Butylgruppen allgemein zur Steigerung der Löslichkeit in organischen Lösungsmitteln führt (vgl. auch ${ }^{19,20)}$ ). Um dieses Prinzip auf 1 anzuwenden ohne den Grundchromophor wesentlich zu stören, sollte $\mathbf{R}$ in 1 ein mit tert-Butyl-Gruppen versehener aromatischer Rest sein. Weiterhin ist zu untersuchen, ob auch mit geeigneten rein aliphatischen Resten R genügend lösliche Farbstoffe erhalten werden können.

\section{Ergebnisse}

Die Perylenfarbstoffe 1 werden durch Kondensation von primären Aminen mit $3,4,9,10$-Perylentetracarbonsäuredianhydrid bei Temperaturen von 180 bis $230^{\circ} \mathrm{C}$ erhalten, wobei Zinkacetat ${ }^{10}$ ) oder Dicyclohexylcarbodiimid als wasserabspaltende Mittel Verwendung finden. Letzteres liefert 1 zwar in geringeren Ausbeuten als Zinkacetat, führt jedoch bei empfindlichen Aminen, wie z. B. 1-Aminoadamantan, weniger leicht zu Zersetzung. Die Reindarstellung von 1 erfolgt durch Behandeln des rohen Reaktionsproduktes mit Natriumcarbonatlösung zum Entfernen von unumgesetztem Anhydrid und anschließend durch Chromatographie oder Kristallisation. Ein gutes Rein- 
heitskriterium für 1 ist seine Feststoff-Fluoreszenz, die bei hochreinen Produkten bereits im Tageslicht leuchtend rot ist. Die spektroskopischen Eigenschaften der dargestellten Perylenfarbstoffe sind Tab. $1 \mathrm{zu}$ entnehmen.<smiles>[R]N1C(=O)c2ccc3c4ccc5c6c(ccc(c7ccc(c2c37)C1=O)c64)C(=O)N([R])C5=O</smiles>

$\stackrel{1}{=}$

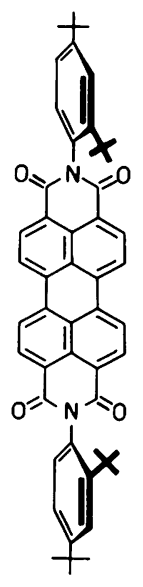

$\stackrel{1}{1, R 8}$

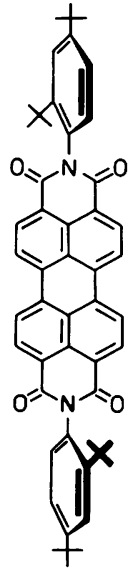

$\stackrel{1}{=}, R 9$

Die Amine zur Darstellung von 1 wurden nach Lit.-Verfahren erhalten.

\section{Diskussion der Ergebnisse}

Alle in Tab. 1 aufgeführten Farbstoffe zeigen nahezu übereinstimmende, stark strukturierte UV/VIS-Absorptionsspektren, bei denen die Schwingungsteilbanden innerhalb von $3 \mathrm{~nm}$ bei $\lambda_{\max }(\lg \varepsilon)=525(4.7), 489$ (4.5), 458 (4.1) nm liegen. Die Spektren werden nur wenig vom Lösungsmittel beeinflußt. Die Fluoreszenzspektren mit Teilbanden

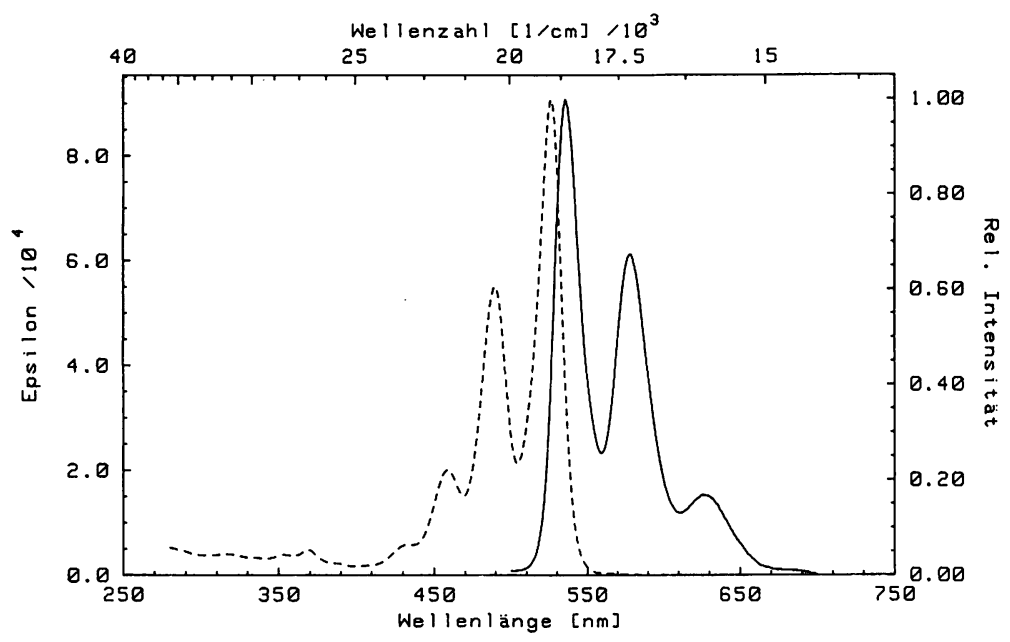

Abb. 1. Korrigiertes UV/VIS-Absorptions- und Fluoreszenz-Spektrum von Farbstoff 1, R 10, in Chloroform 
Tab. 1. Spektroskopische Daten der Perylenfarbstoffe 1

\begin{tabular}{|c|c|c|c|c|c|}
\hline Nr. & Rest $R^{a)}$ & $\varnothing^{b)}$ & $T_{1 / 2}{ }^{c}$ & $\operatorname{MS}[m / e(\%)]^{\mathrm{d})}$ & $C_{\mathrm{s}}{ }^{\mathrm{e})}$ \\
\hline R 1 & Phenyl & 0.93 & 24 & $542\left(100 \%, \mathrm{M}^{+}\right)$ & 4 \\
\hline $\mathrm{R} 2$ & 2,6-Dimethylphenyl & 0.98 & 9.9 & $598\left(100 \%, \mathrm{M}^{+}\right), 581(95 \%, \mathrm{M}-\mathrm{OH})$ & 40 \\
\hline R 3 & 3,5-Dimethylphenyl & 0.78 & - & $\left.598\left(\mathrm{M}^{+}\right)^{\mathrm{g}}\right)$ & - \\
\hline R 4 & 2,4,6-Trimethylphenyl & 0.92 & 8.7 & $626\left(100 \%, M^{+}\right), 609(68 \%, M-O H)$ & 67 \\
\hline R 5 & 2-tert-Butylphenyl $\mathrm{h}^{\mathrm{h}}$ & 0.94 & 16 & $\begin{array}{l}654\left(9 \%, \mathrm{M}^{+}\right), 639\left(6 \%, \mathrm{M}-\mathrm{CH}_{3}\right) \\
637(3 \%, \mathrm{M}-\mathrm{OH}), 597\left(100 \%, \mathrm{M}-\mathrm{C}_{4} \mathrm{H}_{9}\right)\end{array}$ & $60^{\mathrm{h})}$ \\
\hline R 6 & 3-tert-Butylphenyl h) & 1.00 & 33 & $654\left(60 \%, \mathrm{M}^{+}\right), 639\left(100 \%, \mathrm{M}-\mathrm{CH}_{3}\right)$ & 1 \\
\hline R 7 & 4-tert-Butylphenyl & 0.88 & 28 & $\begin{array}{l}654\left(59 \%, \mathrm{M}^{+}\right), 639\left(100 \%, \mathrm{M}-\mathrm{CH}_{3}\right) \\
597\left(3 \%, \mathrm{M}-\mathrm{C}_{4} \mathrm{H}_{9}\right)\end{array}$ & 2 \\
\hline R 8 & cis-2,4-Di-tert-butylphenyl ${ }^{f}$ & 1.00 & 18 & $\begin{array}{l}766\left(3 \%, \mathrm{M}^{+}\right), 751\left(2 \%, \mathrm{M}-\mathrm{CH}_{3}\right) \\
749(2 \%, \mathrm{M}-\mathrm{OH}), 709\left(100 \%, \mathrm{M}-\mathrm{C}_{4} \mathrm{H}_{9}\right)\end{array}$ & 690 \\
\hline R 9 & trans-2,4-Di-tert-butylphenyl ${ }^{\text {f }}$ & 0.98 & 16 & $\begin{array}{l}766\left(3 \%, \mathrm{M}^{+}\right), 751\left(3 \%, \mathrm{M}-\mathrm{CH}_{3}\right) \\
749(2 \%, \mathrm{M}-\mathrm{OH}), 709\left(100 \%, \mathrm{M}-\mathrm{C}_{4} \mathrm{H}_{9}\right)\end{array}$ & 48 \\
\hline R 10 & 2,5-Di-tert-butylphenyl h) & 0.99 & 19 & $\begin{array}{l}766\left(3 \%, \mathrm{M}^{+}\right), 751\left(3 \%, \mathrm{M}-\mathrm{CH}_{3}\right) \\
749(2 \%, \mathrm{M}-\mathrm{OH}), 709\left(100 \%, \mathrm{M}-\mathrm{C}_{4} \mathrm{H}_{9}\right)\end{array}$ & $46^{\mathrm{h})}$ \\
\hline R 11 & 3,5-Di-tert-butylphenyl & 0.96 & 37 & $\left.766\left(M^{+}\right) g\right)$ & 0.2 \\
\hline R 12 & 4-tert-Butyl-2,6-dimethylphenyl h) & 0.97 & 9.1 & $\begin{array}{l}710\left(100 \%, \mathrm{M}^{+}\right), 695\left(71 \%, \mathrm{M}-\mathrm{CH}_{3}\right) \\
693(12 \%, \mathrm{M}-\mathrm{OH}), 653\left(3 \%, \mathrm{M}-\mathrm{C}_{4} \mathrm{H}_{9}\right)\end{array}$ & 51 \\
\hline $\mathrm{R} 13$ & 4,6-di-tert-Butyl-2-methylphenyl h) & 0.87 & 6.8 & $\begin{array}{l}794\left(20 \%, \mathrm{M}^{+}\right), 779\left(5 \%, \mathrm{M}-\mathrm{CH}_{3}\right) \\
737\left(100 \%, \mathrm{M}-\mathrm{C}_{4} \mathrm{H}_{9}\right)\end{array}$ & 250 \\
\hline R 14 & 2-Trifluormethylphenyl ${ }^{\mathrm{h}}$ ) & 0.97 & 7.5 & $678\left(100 \%, \mathrm{M}^{+}\right), 609\left(52 \%, \mathrm{M}-\mathrm{CF}_{3}\right)$ & - \\
\hline $\mathrm{R} 15$ & 3-Trifluormethylphenyl h) & 0.99 & 12 & $678\left(100 \%, \mathrm{M}^{+}\right)$ & - \\
\hline R 16 & 3,5-Bis(trifluormethyl)phenyl & 0.96 & 5.7 & $\left.814\left(M^{+}\right)^{g}\right)$ & - \\
\hline $\mathrm{R} 17$ & 2-Methyl-5-nitrophenyl h) & 0.96 & 1.3 & - & - \\
\hline $\mathrm{R} 18$ & 2-Methyl-6-nitrophenyl h) & 0.89 & 5.5 & $\begin{array}{l}660\left(78 \%, \mathrm{M}^{+}\right), 614\left(100 \%, \mathrm{M}-\mathrm{NO}_{2}\right) \\
568\left(16 \%, \mathrm{M}-2 \mathrm{NO}_{2}\right)\end{array}$ & - \\
\hline R 19 & 2,6-Difluorphenyl h) & - & 1.1 & $614\left(100 \%, \mathrm{M}^{+}\right), 595(35 \%, \mathrm{M}-\mathrm{F})$ & - \\
\hline R 20 & 2,3,6-Tribromphenyl & 0.95 & $\equiv 1.0$ & - & - \\
\hline R 21 & 4-Methoxy-2-methylphenyl h) & 0.03 & - & $\begin{array}{l}630\left(34 \%, \mathrm{M}^{+}\right), 613(100 \%, \mathrm{M}-\mathrm{OH}) \\
599\left(4 \%, \mathrm{M}-\mathrm{OCH}_{3}\right)\end{array}$ & - \\
\hline
\end{tabular}


Tab. 1 (Fortsetzung)

\begin{tabular}{|c|c|c|c|c|c|}
\hline Nr. & Rest Ra) & $\varnothing^{b)}$ & $T_{1 / 2}{ }^{\mathrm{c}}$ & $\mathrm{MS}[m / e(\%)]^{\mathrm{d})}$ & $C_{\mathrm{s}}^{\mathrm{e})}$ \\
\hline R 22 & 2-Hydroxyphenyl h) & 0.02 & 750 & $\begin{array}{l}574\left(51 \%, \mathrm{M}^{+}\right), 557(100 \%, \mathrm{M}-\mathrm{OH}) \\
540(28 \%, \mathrm{M}-2 \mathrm{OH})\end{array}$ & - \\
\hline R 23 & $\begin{array}{l}\text { 5-(Dimethylsulfonamido)-2-methyl- } \\
\text { phenyl } \mathrm{h}^{\mathrm{h}}\end{array}$ & 0.97 & 9.4 & $784\left(4 \%, \mathrm{M}^{+}\right), 677\left(100 \%, \mathrm{M}-\mathrm{SO}_{2} \mathrm{NC}_{2} \mathrm{H}_{5}\right)$ & - \\
\hline R 24 & 3-Sulfonamidophenyl ${ }^{h)}$ & 0.97 & - & - & - \\
\hline R 25 & 2-[2-(Dimethylamino)ethyl]phenyl ${ }^{\mathrm{h})}$ & 0.67 & - & $\left.685\left(M^{+}\right)^{g}\right)$ & - \\
\hline R 26 & 4-[2-(Dimethylamino)ethyl]phenyl & 0.40 & - & - & - \\
\hline R 27 & Cyclobutyl & 0.99 & 38 & $\left.498\left(M^{+}\right) 8\right)$ & - \\
\hline R 28 & Cyclopentyl & 1.00 & 36 & $\left.526\left(\mathrm{M}^{+}\right)^{8}\right)$ & - \\
\hline R 29 & Cyclohexyl & 1.00 & 37 & $\begin{array}{l}554\left(53 \%, \mathrm{M}^{+}\right), 472\left(21 \%, \mathrm{M}-\mathrm{C}_{6} \mathrm{H}_{10}\right) \\
390\left(100 \%, \mathrm{M}-2 \mathrm{C}_{6} \mathrm{H}_{10}\right)\end{array}$ & - \\
\hline R 30 & Cycloheptyl & 0.98 & 44 & $582\left(\mathrm{M}^{+}\right)^{\mathrm{g})}$ & - \\
\hline R 31 & Cyclooctyl & 0.65 & - & $\begin{array}{l}610\left(4 \%, \mathrm{M}^{+}\right), 500\left(11 \%, \mathrm{M}-\mathrm{C}_{8} \mathrm{H}_{14}\right) \\
390\left(100 \%, \mathrm{M}-2 \mathrm{C}_{8} \mathrm{H}_{14}\right)\end{array}$ & - \\
\hline R 32 & Cyclododecyl & 0.99 & 36 & $722\left(3 \%, \mathrm{M}^{+}\right), 390\left(100 \%, \mathrm{M}-2 \mathrm{C}_{12} \mathrm{H}_{22}\right)$ & - \\
\hline R 33 & 1-Methylcyclopentyl & 1.00 & 47 & $\begin{array}{l}554\left(20 \%, \mathrm{M}^{+}\right), 472\left(24 \%, \mathrm{M}-\mathrm{C}_{6} \mathrm{H}_{10}\right) \\
390\left(100 \%, \mathrm{M}-2 \mathrm{C}_{6} \mathrm{H}_{10}\right)\end{array}$ & \\
\hline R 34 & 2-Hydroxycyclohexyl & 0.99 & 33 & $\begin{array}{l}586\left(4 \%, \mathrm{M}^{+}\right), 568\left(2 \%, \mathrm{M}-\mathrm{H}_{2} \mathrm{O}\right) \\
550\left(4 \%, \mathrm{M}-2 \mathrm{H}_{2} \mathrm{O}\right), 391(100 \%)\end{array}$ & - \\
\hline R 35 & 4-tert-Butylcyclohexyl & 0.97 & 53 & - & - \\
\hline R 36 & 1-Adamantyl & 0.98 & - & - & - \\
\hline R 37 & 1-Bicyclo[2.2.2]octyl & 0.95 & - & $606\left(100 \%, M^{+}\right)$ & - \\
\hline R 38 & 1-Norbornyl & 0.96 & 85 & $578\left(100 \%, \mathrm{M}^{+}\right)$ & - \\
\hline
\end{tabular}


bei 540 und $575 \mathrm{~nm}$ stimmen bei verschiedenen Farbstoffen innerhalb von $5 \mathrm{~nm}$ überein. Abb. 1 zeigt das typische Spektrum von Farbstoff 1, R 10 (s. Tab. 1), in Chloroform. Mit einer durchschnittlichen Trennzahl ${ }^{12)} T z$ von 1.5 liegt jedoch eine weitgehende Überlappung von Absorptions- und Fluoreszenzspektrum vor.

Bei Substitution von $\mathbf{R}=$ Phenyl in 1 mit tert-Butyl-Gruppen wird eine Löslichkeitssteigerung in DMF bis zu einem Faktor von 170 beobachtet (Tab. 1, Nr. R 8 im Vergleich zu R 1). Wie z. B. die beiden atropisomeren Farbstoffe R 8 und R 9 zeigen, deren Trennung chromatographisch möglich ist, ist jedoch die Löslichkeits-steigernde Wirkung der Substituenten stark von deren räumlicher Orientierung abhängig. Dies könnte einmal mit Kristallgitter-Packungseffekten und zum anderen mit den unterschiedlichen Dipolmomenten der Farbstoffe in Zusammenhang stehen. Bemerkenswert ist, daß der symmetrisch substituierte Farbstoff R 11 sogar eine geringere Löslichkeit als R 1 besitzt. Farbstoffe mit rein aliphatischen Resten $R$ (Tab. 1, R 27 bis R 38) zeigen i. allg. eine Löslichkeit, die um ein bis zwei Zehnerpotenzen geringer ist als mit $R=$ Aryl. Verhältnismäßig gut sind noch die Farbstoffe R 28 und R 32 löslich, die Cyclopentyl- und Cyclododecylgruppen tragen; extrem schwerlöslich ist dagegen der Farbstoff $R 31$ mit Cyclooctylgruppen. Die anderen Farbstoffe mit rein aliphatischen Resten nehmen eine Mittelstellung zwischen diesen Extremen ein. Die in DMF beobachtete Löslichkeitsabstufung der Farbstoffe wird auch in Toluol, Pivalinsäure-methylester und in polymeren Gläsern wie z. B. Polymethylmethacrylat beobachtet.

Bei einem Vergleich der Fluoreszenzquantenausbeuten in Tab. 1 fällt auf, daß der bereits hohe Wert von ca. $90 \%$ für $\mathrm{R}=$ Phenyl bis auf $100 \%$ steigt, wenn $\mathrm{R}$ in orthoPosition voluminöse Gruppen trägt. Dies legt nahe, daß ein wichtiger strahlungsloser Desaktivierungsweg die Anregung einer Rotation oder Torsionsschwingung um die $\mathrm{R}-\mathrm{N}$-Bindung in 1 ist (vgl. auch Lit. ${ }^{13)}$ ). Eine Besonderheit von $\mathbf{1}$ ist, daß $\mathrm{NO}_{2}$ - oder Br-Gruppen nicht zu einer Fluoreszenzlöschung über Prädissoziationsprozesse führen (R 17, R 18 und R 20). Methoxy- oder Hydroxygruppen bewirken jedoch weitgehende Fluoreszenzlöschung ( 21 und R 22). Farbstoffe mit $R=$ Alkyl fluoreszieren durchweg mit hohen Quantenausbeuten.

Die relativen Photostabilitäten der Farbstoffe, die nur bei extrem hohen Lichtintensitäten bestimmt werden können, sind als relative Halbwertszeiten bei Bestrahlung Tab.1 zu entnehmen. Die Photobleichvorgänge von 1 im Lösungsmittel DMF folgen bei konstanter, hoher Lichtintensität streng dem Zeitgesetz der 1 . Ordnung $(1 \mathrm{~cm}$ Schichtdicke, Beginn mit $\mathrm{E}_{\lambda_{\max }}=1.0$ ). Die hieraus erhaltenen Geschwindigkeitskonstanten bzw. Halbwertszeiten sind ein Maß für Quantenausbeuten von Photobleichreaktionen. Es wird dabei gefunden, daß Farbstoffe mit aromatischen Resten $R$, die benzylständige Wasserstoffe tragen, verhältnismäßig schnell ausbleichen (z. B. R 2, R 4, R 12 und R 17). Daher kann geschlossen werden, daß ein oxidativer Abbau über Radikalreaktionen, die in Benzylstellung bekanntlich besonders leicht erfolgen, oder über En-Reaktionen eine wichtige Stellung bei den Photobleichreaktionen einnimmt. Trägt jedoch der aromatische Rest $\mathrm{R}$ nur tert-Alkylgruppen, sind die Farbstoffe wesentlich photostabiler (z. B. R 5, R 6, R 7, R 10 und R 11). Überraschend ist die geringe Beständigkeit der Farbstoffe, wenn $R$ mit Halogenen oder Trifluormethylgruppen substituiert ist (z. B. R 19, R 20 und R 16). Dies ist sogar bei einer Substitution mit Fluor trotz der großen Bindungsdissoziationsenergie der $\mathrm{C}-\mathrm{F}$-Bindung der Fall und spricht

Chem. Ber. 115 (1982) 
daher auch für eine Beteiligung ionischer Prozesse an den Photobleichreaktionen. Farbstoffe mit $\mathbf{R}=$ Alkyl weisen durchweg eine größere Beständigkeit auf als mit $\mathbf{R}=$ Aryl. Farbstoffe mit tertiären Alkylresten $\mathrm{R}$ sind stabiler als solche mit sekundären (vgl. R 28 mit R 33).

Der photostabilste Fluoreszenzfarbstoff 1 überhaupt ist Nr. R 38 mit R = 1-Norbornyl (R 22 zeigt nur verschwindend geringe Fluoreszenz). Dies steht damit im Einklang, daß durch Substitution mit dem starren Brückenkopfsystem ionische ebenso wie radikalische Abbaureaktionen und auch Eliminierungen erschwert sind.

Für einen praktischen Einsatz der Fluoreszenzfarbstoffe 1 sind Nr. R 10 und R 32 besonders geeignet, da sie einen Kompromiß bieten zwischen großer Fluoreszenzquantenausbeute, Löslichkeit, Photostabilität und leichter Zugänglichkeit.

Der Deutschen Forschungsgemeinschaft und dem Bundesministerium für Forschung und Technologie (Projekt ET 4428 A), sowie den Herren Prof. Dr. C. Rüchardt und Prof. Dr. A. Götzberger wird für die Unterstützung der Arbeit gedankt. Das korrigierte UV/VIS-Absorptions- und Fluoreszenzspektrum wurde freundlicherweise von Herrn Prof. Dr. W. Lüttke zur Verfügung gestellt. Der BASF Aktiengesellschaft sei für die Spende von 3,4,9,10-Perylentetracarbonsäuredianhydrid gedankt.

\section{Experimenteller Teil}

Massenspektren: SM 1 B, MAT 312 (Fa. Varian) und MAT 312 (Fa. Finnigen); UV/VIS-Spektren: DMR 21, PMQ 3 (Fa. Zeiss).

3,4,9,10-Perylentetracarbonsäurediimide 1: $1.0 \mathrm{~g}$ (2.5 mmol) 3,4,9,10-Perylentetracarbonsäuredianhydrid wird unter Stickstoff-Schutzatmosphäre unter Zusatz von $350 \mathrm{mg}(1.9 \mathrm{mmol}) \mathrm{ge}-$ trocknetem Zinkacetat (Methode A) ${ }^{10)}$ bzw. $350 \mathrm{mg}(1.7 \mathrm{mmol})$ Dicyclohexylcarbodiimid (Methode B) und $5 \mathrm{ml}$ frisch destilliertem Chinolin $1-2 \mathrm{~h}$ bei $180-230^{\circ} \mathrm{C}$ mit $10 \mathrm{mmol}$ eines primären Amins umgesetzt. Das Kondensationsprodukt wird zur Entfernung von unumgesetztem Edukt mehrfach mit jeweils $112 \mathrm{proz} . \mathrm{Na}_{2} \mathrm{CO}_{3}$-Lösung ausgekocht. Die weitere Reinigung erfolgt durch Umkristallisieren (Toluol, Xylol, o-Dichlorbenzol oder DMF) oder Chromatographie (Aluminiumoxid neutral mit DMF oder Toluol/Aceton 4:1 bzw. Kieselgel mit Methylenchlorid). Reine Produkte können auch durch Sublimation im Hochvakuum erhalten werden.

Ausbeuten und physikalische Eigenschaften der Farbstoffe siehe Tab. 2.

Bestimmung der Photostabilitäten von 1: Zur Erzielung einer genügend hohen Strahlungsdichte im sichtbaren Bereich des Spektrums wird das Licht der Natrium-, Indium- und Thallium-Entladungslampe HQI-T $2000 \mathrm{~W} / \mathrm{N}$ der Fa. Osram mit Hilfe eines linearen Ellipsenspiegels (Halbachsen: $a=30, b=25.95 \mathrm{~cm}$, lineare Exzentrizität $\varepsilon=15 \mathrm{~cm}$, Länge $l$ in Richtung der Halbachse $a: 41.7 \mathrm{~cm}$ ) auf eine Fläche von ca. $2 \times 40 \mathrm{~cm}$ fokussiert. In der Brennebene dieser Anordnung wird in einem thermostatisierbaren Küvettenhalter die zu untersuchende Farbstofflösung mit $E_{\lambda_{\max }}=1.0$ in Glasküvetten von $1 \mathrm{~cm}$ Schichtdicke unter Zutritt von Luft bestrahlt. Zu genau definierten Zeiten wird die Bestrahlung unterbrochen und die Restkonzentration an Farbstoff UV/VIS-spektroskopisch bestimmt (über ca. $2 \frac{1}{2}$ Halbwertszeiten). Die Geschwindigkeitskonstanten der Photobleichreaktionen werden wie üblich aus den Extinktionswerten berechnet. Die Photobleichreaktion erfolgt bis über 2 Halbwertszeiten streng nach 1. Ordnung (Korrelationskoeffizient $>0.999$ bei 10 Meßwerten). Die Endextinktion der Lösung ist bei fast allen Farbstoffen nach 10-15. Halbwertszeiten verschwindend gering. 
Tab. 2. Ausbeuten, physikalische Daten und Elementaranalysen der Perylenfarbstoffe 1

\begin{tabular}{|c|c|c|c|c|c|c|c|c|c|}
\hline \multirow{3}{*}{$\begin{array}{l}\text { Nr. }{ }^{a)} \\
\text { R } 1\end{array}$} & \multirow{3}{*}{$\begin{array}{c}\begin{array}{c}\text { Darstel- } \\
\text { lung }{ }^{\text {) }}\end{array} \\
\mathrm{A}\end{array}$} & \multirow{3}{*}{$\begin{array}{c}\begin{array}{c}\text { Ausb.c) } \\
(\%)\end{array} \\
98\end{array}$} & \multirow{3}{*}{$\begin{array}{l}R_{\mathrm{F}}^{\mathrm{d}, \mathrm{e})} \\
0.50\end{array}$} & \multirow{3}{*}{$\begin{array}{c}\begin{array}{c}\text { Summenformel } \\
\text { (Molmasse) }\end{array} \\
\mathrm{C}_{36} \mathrm{H}_{18} \mathrm{~N}_{2} \mathrm{O}_{4} \\
(542.6)\end{array}$} & \multicolumn{3}{|c|}{ Elementaranalyse } & & \multirow{2}{*}{ Lit. } \\
\hline & & & & & C & $\mathrm{H}$ & $N$ & & \\
\hline & & & & & $\begin{array}{l}\text { Ber. } 79.70 \\
\text { Gef. } 79.79\end{array}$ & $\begin{array}{l}3.34 \\
3.45\end{array}$ & $\begin{array}{l}5.16 \\
4.85\end{array}$ & & 14) \\
\hline R 2 & A & 78 & 0.13 & $\begin{array}{c}\mathrm{C}_{40} \mathrm{H}_{26} \mathrm{~N}_{2} \mathrm{O}_{4} \\
(598.7)\end{array}$ & $\begin{array}{l}\text { Ber. } 80.25 \\
\text { Gef. } 80.33\end{array}$ & $\begin{array}{l}4.38 \\
4.43\end{array}$ & $\begin{array}{l}4.68 \\
4.75\end{array}$ & & 10) \\
\hline R 3 & A & 98 & 0.47 & $\begin{array}{c}\mathrm{C}_{40} \mathrm{H}_{26} \mathrm{~N}_{2} \mathrm{O}_{4} \\
(598.7)\end{array}$ & $\begin{array}{l}\text { Ber. } 80.25 \\
\text { Gef. } 80.42\end{array}$ & $\begin{array}{l}4.38 \\
4.41\end{array}$ & $\begin{array}{l}4.68 \\
4.51\end{array}$ & & 15) \\
\hline R 4 & A & 67 & 0.25 & $\begin{array}{c}\mathrm{C}_{32} \mathrm{H}_{30} \mathrm{~N}_{2} \mathrm{O}_{4} \\
(626.7)\end{array}$ & $\begin{array}{l}\text { Ber. } 80.49 \\
\text { Gef. } 80.31\end{array}$ & $\begin{array}{l}4.83 \\
4.88\end{array}$ & $\begin{array}{l}4.47 \\
4.46\end{array}$ & & 10) \\
\hline R 5 & A & 71 & $\begin{array}{l}0.17 \text { u. } \\
0.34\end{array}$ & $\begin{array}{c}\mathrm{C}_{44} \mathrm{H}_{34} \mathrm{~N}_{2} \mathrm{O}_{4} \\
(654.8)\end{array}$ & $\begin{array}{l}\text { Ber. } 80.71 \\
\text { Gef. } 80.57\end{array}$ & $\begin{array}{l}5.23 \\
5.30\end{array}$ & $\begin{array}{l}4.28 \\
4.06\end{array}$ & & - \\
\hline R 6 & A & 98 & 0.17 & $\begin{array}{c}\mathrm{C}_{44} \mathrm{H}_{34} \mathrm{~N}_{2} \mathrm{O}_{4} \\
(654.8)\end{array}$ & $\begin{array}{l}\text { Ber. } 80.71 \\
\text { Gef. } 80.10\end{array}$ & $\begin{array}{l}5.23 \\
5.22\end{array}$ & $\begin{array}{l}4.28 \\
4.46\end{array}$ & & - \\
\hline R 7 & A & 98 & 0.63 & $\begin{array}{c}\mathrm{C}_{44} \mathrm{H}_{34} \mathrm{~N}_{2} \mathrm{O}_{4} \\
(654.8)\end{array}$ & $\begin{array}{l}\text { Ber. } 80.71 \\
\text { Gef. } 80.59\end{array}$ & $\begin{array}{l}5.23 \\
5.15\end{array}$ & $\begin{array}{l}4.28 \\
4.08\end{array}$ & & - \\
\hline R 8 & A & $65^{f)}$ & 0.24 & $\begin{array}{c}\mathrm{C}_{52} \mathrm{H}_{50} \mathrm{~N}_{2} \mathrm{O}_{4} \\
(767.0)\end{array}$ & $\begin{array}{l}\text { Ber. } 81.43 \\
\text { Gef. } 81.39\end{array}$ & $\begin{array}{l}6.57 \\
6.52\end{array}$ & $\begin{array}{l}3.65 \\
3.81\end{array}$ & & - \\
\hline R 9 & A & $65^{f)}$ & 0.52 & $\begin{array}{c}\mathrm{C}_{52} \mathrm{H}_{50} \mathrm{~N}_{2} \mathrm{O}_{4} \\
(767.0)\end{array}$ & $\begin{array}{l}\text { Ber. } 81.43 \\
\text { Gef. } 81.20\end{array}$ & $\begin{array}{l}6.57 \\
6.53\end{array}$ & $\begin{array}{l}3.65 \\
3.82\end{array}$ & & - \\
\hline R 10 & A & 89 & 0.42 & $\begin{array}{c}\mathrm{C}_{52} \mathrm{H}_{50} \mathrm{~N}_{2} \mathrm{O}_{4} \\
(767.0)\end{array}$ & $\begin{array}{l}\text { Ber. } 81.43 \\
\text { Gef. } 81.62\end{array}$ & $\begin{array}{l}6.57 \\
6.66\end{array}$ & $\begin{array}{l}3.65 \\
3.97\end{array}$ & & - \\
\hline R 11 & A & 84 & 0.52 & $\begin{array}{c}\mathrm{C}_{52} \mathrm{H}_{50} \mathrm{~N}_{2} \mathrm{O}_{4} \\
(767.0)\end{array}$ & $\begin{array}{l}\text { Ber. } 81.43 \\
\text { Gef. } 80.71\end{array}$ & $\begin{array}{l}6.57 \\
6.62\end{array}$ & $\begin{array}{l}3.65 \\
3.50\end{array}$ & & - \\
\hline R 12 & A & 86 & 0.43 & $\begin{array}{c}\mathrm{C}_{48} \mathrm{H}_{42} \mathrm{~N}_{2} \mathrm{O}_{4} \\
(710.9)\end{array}$ & $\begin{array}{l}\text { Ber. } 81.10 \\
\text { Gef. } 80.84\end{array}$ & $\begin{array}{l}5.96 \\
6.21\end{array}$ & $\begin{array}{l}3.94 \\
3.90\end{array}$ & & - \\
\hline R 13 & A & 42 & 0.49 & $\begin{array}{l}\mathrm{C}_{54} \mathrm{H}_{54} \mathrm{~N}_{2} \mathrm{O}_{4} \\
(795.0)\end{array}$ & $\begin{array}{l}\text { Ber. } 81.58 \\
\text { Gef. } 81.74\end{array}$ & $\begin{array}{l}6.85 \\
6.91\end{array}$ & $\begin{array}{l}3.52 \\
3.25\end{array}$ & & - \\
\hline R 14 & A & 88 & 0.54 & $\begin{array}{c}\mathrm{C}_{38} \mathrm{H}_{16} \mathrm{~F}_{6} \mathrm{~N}_{2} \mathrm{O}_{4} \\
(678.6)\end{array}$ & $\begin{array}{l}\text { Ber. } \\
\text { Gef. }\end{array}$ & & $\begin{array}{l}4.13 \\
4.12\end{array}$ & & - \\
\hline R 15 & A & 83 & 0.67 & $\begin{array}{l}\mathrm{C}_{38} \mathrm{H}_{16} \mathrm{~F}_{6} \mathrm{~N}_{2} \mathrm{O}_{4} \\
\quad(678.6)\end{array}$ & $\begin{array}{l}\text { Ber. } 67.26 \\
\text { Gef. } 67.29\end{array}$ & $\begin{array}{l}2.38 \\
2.63\end{array}$ & $\begin{array}{l}4.13 \\
4.37\end{array}$ & & 18) \\
\hline R 16 & A & 85 & 0.56 & $\begin{array}{c}\mathrm{C}_{40} \mathrm{H}_{14} \mathrm{~F}_{12} \mathrm{~N}_{2} \mathrm{O}_{4} \\
(814.5)\end{array}$ & $\begin{array}{l}\text { Ber. } \\
\text { Gef. }\end{array}$ & & $\begin{array}{l}3.44 \\
3.47\end{array}$ & & - \\
\hline R 17 & A & 85 & 0.44 & $\begin{array}{l}\mathrm{C}_{38} \mathrm{H}_{20} \mathrm{~N}_{4} \mathrm{O}_{8} \\
(660.6)\end{array}$ & $\begin{array}{l}\text { Ber. } 69.09 \\
\text { Gef. } 69.55\end{array}$ & $\begin{array}{l}3.05 \\
3.20\end{array}$ & $\begin{array}{l}8.48 \\
8.56\end{array}$ & & - \\
\hline R 18 & A & 98 & 0.42 & $\begin{array}{c}\mathrm{C}_{38} \mathrm{H}_{20} \mathrm{~N}_{4} \mathrm{O}_{8} \\
(660.6)\end{array}$ & $\begin{array}{l}\text { Ber. } 69.09 \\
\text { Gef. } 68.62\end{array}$ & $\begin{array}{l}3.05 \\
3.41\end{array}$ & $\begin{array}{l}8.48 \\
8.44\end{array}$ & & - \\
\hline R 19 & A & 97 & 0.24 & $\begin{array}{l}\mathrm{C}_{36} \mathrm{H}_{14} \mathrm{~F}_{4} \mathrm{~N}_{2} \mathrm{O}_{4} \\
(614.5)\end{array}$ & $\begin{array}{l}\text { Ber. } 70.36 \\
\text { Gef. } 69.30\end{array}$ & $\begin{array}{l}2.30 \\
2.38\end{array}$ & $\begin{array}{l}4.56 \\
4.99\end{array}$ & & - \\
\hline R 20 & A & 98 & 0.48 & $\begin{array}{c}\mathrm{C}_{36} \mathrm{H}_{12} \mathrm{Br}_{6} \mathrm{~N}_{2} \mathrm{O}_{4} \\
\quad(1015.9)\end{array}$ & $\begin{array}{l}\text { Ber. } 42.56 \\
\text { Gef. } 42.99\end{array}$ & $\begin{array}{l}1.19 \\
1.20\end{array}$ & $\begin{array}{l}2.76 \\
2.97\end{array}$ & $\begin{array}{l}\text { Br } 47.19 \\
\text { Br } 46.64\end{array}$ & - \\
\hline R 21 & A & 95 & 0.39 & $\begin{array}{c}\mathrm{C}_{40} \mathrm{H}_{26} \mathrm{~N}_{2} \mathrm{O}_{6} \\
(630.7)\end{array}$ & $\begin{array}{l}\text { Ber. } 76.18 \\
\text { Gef. } 76.43\end{array}$ & $\begin{array}{l}4.16 \\
4.23\end{array}$ & $\begin{array}{l}4.44 \\
4.45\end{array}$ & & - \\
\hline R 22 & A & 95 & 0.06 & - & - & & & & - \\
\hline R 23 & A & 70 & 0.10 & $\begin{array}{l}\mathrm{C}_{42} \mathrm{H}_{32} \mathrm{~N}_{4} \mathrm{O}_{8} \mathrm{~S}_{2} \\
\quad(784.9)\end{array}$ & $\begin{array}{l}\text { Ber. } 64.27 \\
\text { Gef. } 64.15\end{array}$ & $\begin{array}{l}4.11 \\
4.31\end{array}$ & $\begin{array}{l}7.14 \\
7.18\end{array}$ & $\begin{array}{ll}\text { S } & 8.17 \\
\text { S } & 7.96\end{array}$ & 17) \\
\hline R 24 & A & 91 & 0.15 & $\begin{array}{l}\mathrm{C}_{36} \mathrm{H}_{20} \mathrm{~N}_{4} \mathrm{O}_{8} \mathrm{~S}_{2} \\
\quad(700.7)\end{array}$ & $\begin{array}{l}\text { Ber. } 61.71 \\
\text { Gef. } 61.53\end{array}$ & $\begin{array}{l}2.88 \\
3.07\end{array}$ & $\begin{array}{l}8.00 \\
8.03\end{array}$ & $\begin{array}{ll}\text { S } & 9.15 \\
\text { S } & 8.44\end{array}$ & - \\
\hline $\begin{array}{l}\text { R } 25 \\
\text { R } 26\end{array}$ & $\begin{array}{l}\mathrm{A} \\
\mathrm{A}\end{array}$ & $\begin{array}{l}49 \\
74\end{array}$ & $\begin{array}{l}0.57 \\
-\end{array}$ & $\begin{array}{l}\overline{\mathrm{C}}_{44} \mathrm{H}_{36} \mathrm{~N}_{4} \mathrm{O}_{4} \\
\quad(684.8)\end{array}$ & $\begin{array}{l}- \\
\text { Ber. } 77.17 \\
\text { Gef. } 77.38\end{array}$ & $\begin{array}{l}5.30 \\
5.18\end{array}$ & $\begin{array}{l}8.18 \\
8.00\end{array}$ & & $\begin{array}{l}- \\
-\end{array}$ \\
\hline
\end{tabular}

Chem. Ber. 115 (1982) 
Tab. 2 (Fortsetzung)

\begin{tabular}{|c|c|c|c|c|c|c|c|c|}
\hline \multirow{2}{*}{$\begin{array}{l}\mathrm{Nr}^{\text {a) }} \\
\text { R } 27\end{array}$} & \multirow{2}{*}{$\frac{\begin{array}{c}\text { Darstel- } \\
\text { lung b) }\end{array}}{\mathrm{A}}$} & \multirow{2}{*}{$\begin{array}{c}\begin{array}{c}\text { Ausb.c) } \\
(\%)\end{array} \\
95\end{array}$} & \multirow{2}{*}{$\begin{array}{l}R_{\mathrm{F}}^{\mathrm{d}, \mathrm{e})} \\
0.30\end{array}$} & \multirow{2}{*}{$\begin{array}{l}\begin{array}{c}\text { Summenformel } \\
\text { (Molmasse) }\end{array} \\
-\end{array}$} & \multicolumn{3}{|c|}{ Elementaranalyse } & Lit. \\
\hline & & & & & _- & & & _- \\
\hline R 28 & A & 91 & 0.50 & $\begin{array}{c}\mathrm{C}_{34} \mathrm{H}_{26} \mathrm{~N}_{2} \mathrm{O}_{4} \\
(526.6)\end{array}$ & $\begin{array}{l}\text { Ber. } 77.55 \\
\text { Gef. } 76.98\end{array}$ & $\begin{array}{l}4.98 \\
4.75\end{array}$ & $\begin{array}{l}5.32 \\
5.31\end{array}$ & - \\
\hline R 29 & A & 98 & 0.57 & $\begin{array}{c}\mathrm{C}_{36} \mathrm{H}_{30} \mathrm{~N}_{2} \mathrm{O}_{4} \\
(554.7)\end{array}$ & $\begin{array}{l}\text { Ber. } 77.96 \\
\text { Gef. } 77.56\end{array}$ & $\begin{array}{l}5.47 \\
5.45\end{array}$ & $\begin{array}{l}5.50 \\
4.95\end{array}$ & 16) \\
\hline R 30 & A & 94 & 0.59 & $\begin{array}{c}\mathrm{C}_{38} \mathrm{H}_{34} \mathrm{~N}_{2} \mathrm{O}_{4} \\
(582.7)\end{array}$ & $\begin{array}{l}\text { Ber. } 78.33 \\
\text { Gef. } 78.35\end{array}$ & $\begin{array}{l}5.88 \\
5.71\end{array}$ & $\begin{array}{l}4.81 \\
4.59\end{array}$ & - \\
\hline R 31 & A & 98 & 0.70 & - & - & & & - \\
\hline R 32 & A & 97 & 0.73 & $\begin{array}{c}\mathrm{C}_{48} \mathrm{H}_{54} \mathrm{~N}_{2} \mathrm{O}_{4} \\
(723.0)\end{array}$ & $\begin{array}{l}\text { Ber. } 79.74 \\
\text { Gef. } 79.56\end{array}$ & $\begin{array}{l}7.53 \\
7.50\end{array}$ & $\begin{array}{l}3.97 \\
4.05\end{array}$ & - \\
\hline R 33 & A & 68 & 0.56 & $\begin{array}{c}\mathrm{C}_{36} \mathrm{H}_{30} \mathrm{~N}_{2} \mathrm{O}_{4} \\
(554.7)\end{array}$ & $\begin{array}{l}\text { Ber. } 77.96 \\
\text { Gef. } 78.12\end{array}$ & $\begin{array}{l}5.45 \\
5.33\end{array}$ & $\begin{array}{l}5.05 \\
5.35\end{array}$ & - \\
\hline R 34 & A & 91 & 0.01 & $\begin{array}{c}\mathrm{C}_{36} \mathrm{H}_{30} \mathrm{~N}_{2} \mathrm{O}_{6} \\
(586.6)\end{array}$ & $\begin{array}{l}\text { Ber. } 73.71 \\
\text { Gef. } 73.83\end{array}$ & $\begin{array}{l}5.15 \\
4.67\end{array}$ & $\begin{array}{l}4.77 \\
5.15\end{array}$ & - \\
\hline R 35 & A & 96 & 0.81 & $\begin{array}{c}\mathrm{C}_{44} \mathrm{H}_{46} \mathrm{~N}_{2} \mathrm{O}_{4} \\
(666.9)\end{array}$ & $\begin{array}{l}\text { Ber. } 79.25 \\
\text { Gef. } 79.36\end{array}$ & $\begin{array}{l}6.95 \\
6.92\end{array}$ & $\begin{array}{l}4.20 \\
4.65\end{array}$ & - \\
\hline R 36 & B & 55 & 0.57 & $\begin{array}{c}\mathrm{C}_{44} \mathrm{H}_{38} \mathrm{~N}_{2} \mathrm{O}_{4} \\
(658.8)\end{array}$ & $\begin{array}{l}\text { Ber. } 80.22 \\
\text { Gef. } 79.16\end{array}$ & $\begin{array}{l}5.81 \\
5.36\end{array}$ & $\begin{array}{l}4.25 \\
4.10\end{array}$ & - \\
\hline R 37 & A & 75 & 0.63 & $\begin{array}{c}\mathrm{C}_{40} \mathrm{H}_{34} \mathrm{~N}_{2} \mathrm{O}_{4} \\
(606.7)\end{array}$ & $\begin{array}{l}\text { Ber. } 79.19 \\
\text { Gef. } 78.40\end{array}$ & $\begin{array}{l}5.65 \\
5.49\end{array}$ & $\begin{array}{l}4.62 \\
4.34\end{array}$ & - \\
\hline R 38 & A & 88 & 0.57 & $\begin{array}{c}\mathrm{C}_{38} \mathrm{H}_{30} \mathrm{~N}_{2} \mathrm{O}_{4} \\
(578.7)\end{array}$ & $\begin{array}{l}\text { Ber. } 78.87 \\
\text { Gef. } 78.33\end{array}$ & $\begin{array}{l}5.23 \\
5.42\end{array}$ & $\begin{array}{l}4.84 \\
5.04\end{array}$ & - \\
\hline
\end{tabular}

a) Siehe Tab. 1. - b) Siehe Synthesevorschrift. - c) Isolierte Ausbeuten des Rohprodukts, Reinheit $>95 \%$. - d) $R_{\mathrm{F}}$-Wert bei Dünnschichtchromatographie: $\mathrm{CHCl}_{3}, \mathrm{Al}_{2} \mathrm{O}_{3}$ (Alox N/UV 254 , Fa. Macherey \& Nagel). - e) Schmelzpunkte aller Farbstoffe $>360^{\circ} \mathrm{C}$. - f) Gemeinsame Ausbeute beider Atropisomerer.

1) A. Rademacher, Dissertation, Univ. Freiburg 1981.

2) S. Märkle, Staatsexamensarbeit, Univ. Freiburg 1980.

3) $H$. Langhals, Nachr. Chem. Tech. 28, 716 (1980).

4) A. Goetzberger und W. Greubel, Appl. Phys. 14, 123 (1977).

5) R. L. Garvin, Rev. Sci. Instrum. 31, 1010 (1960).

6) $H$. R. Schweizer, Künstliche Organische Farbstoffe und ihre Zwischenprodukte, S. 384, Springer-Verlag, Berlin 1964.

7) E. N. Abrahart, Dyes and their Intermediates, 2. Aufl. Edward Arnold, London 1977.

8) H. Langhals, DE $3016764 \mathrm{~A} 1$ vom 30. 4. 1980.

9) $K$. Peters, H. Langhals und G. Werner, Cryst. Struct. Commun. 10, 713 (1981).

10) BASF AG (Erf. F. Graser) D. O. S. 2139688 (15. 2. 1973); D. O. S. 2210170 (6. 9. 1973) [Chem. Abstr. 78, 137962v (1973); Chem. Abstr. 80, 49261b (1974)].

11) A. Zastrow, Dissertation, Univ. Freiburg 1981.

12) $H$. Langhals, Ber. Bunsenges. Phys. Chem. 83, 730 (1979).

13) G. Bott, L. D. Field und S. Sternhell, J. Am. Chem. Soc. 102, 5618 (1980).

14) Sandoz AG, Brit. Pat. 901964 (25. 7. 1962) [Chem. Abstr. 58, p1601b (1963)].

15) Mitsubishi Chemical Industries, Japan. Kokai 7607025 (21. 1. 1976) [Chem. Abstr. 84, p166251s (1976)].

10) Hoechst AG (Erf. G. Geissler und H. Remy), D. B. P. 1130099 (24. 10. 1959) [Chem. Abstr. 57, p11346e (1962)].

17) $B A S F A G$ (Erf. F. Graser), D. O. S. 2147024 (29. 3. 1973) [Chem. Abstr. 79, p6781h (1973)].

18) Nippon Kayaku Co. (Erf. M. Yamada), Japan. Kokai 7637918 (30. 3. 1976) [Chem. Abstr. 85, p34646k (1976)].

19) Geigy, Franz. P. 1580683 [Chem. Abstr. 73, 100057h (1970)].

20) G. Pawlowski und M. Hanack, Synthesis 1980, 287. 